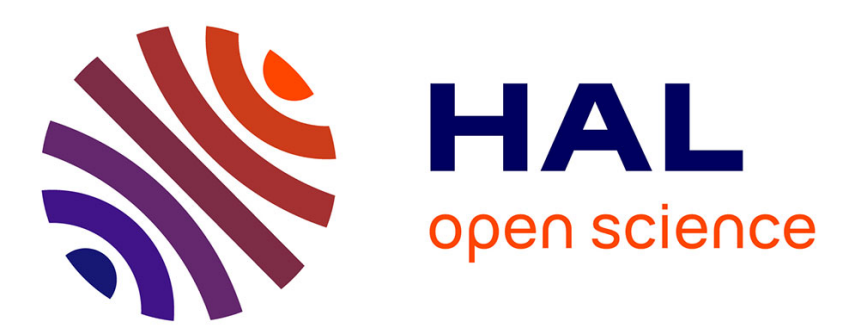

\title{
Photonic orbital angular momentum in turbulence: vortex splitting and adaptive optics
}

\author{
Giacomo Sorelli, Vyaceslav N Shatokhin, Andreas Buchleitner
}

\section{To cite this version:}

Giacomo Sorelli, Vyaceslav N Shatokhin, Andreas Buchleitner. Photonic orbital angular momentum in turbulence: vortex splitting and adaptive optics. SPIE Remote Sensing, Sep 2020, Online Only, France. pp.10, 10.1117/12.2573474 . hal-03267308

\section{HAL Id: hal-03267308 \\ https://hal.sorbonne-universite.fr/hal-03267308}

Submitted on 22 Jun 2021

HAL is a multi-disciplinary open access archive for the deposit and dissemination of scientific research documents, whether they are published or not. The documents may come from teaching and research institutions in France or abroad, or from public or private research centers.
L'archive ouverte pluridisciplinaire HAL, est destinée au dépôt et à la diffusion de documents scientifiques de niveau recherche, publiés ou non, émanant des établissements d'enseignement et de recherche français ou étrangers, des laboratoires publics ou privés. 


\title{
Photonic orbital angular momentum in turbulence: vortex splitting and adaptive optics
}

\author{
Giacomo Sorelli ${ }^{1,2,3}$, Vyaceslav N. Shatokhin ${ }^{4,5}$, Andreas Buchleitner 4,5 \\ ${ }^{1}$ Laboratoire Kastler Brossel, Sorbonne Université, ENS-Université PSL, Collège de France, \\ CNRS; 4 place Jussieu, F-75252 Paris, France \\ 2 DEMR, ONERA, Université Paris Saclay, F-91123, Palaiseau, France \\ 3 Sorbonne Université, CNRS, LIP6, 4 place Jussieu, F-75005 Paris, France \\ 4 Physikalisches Institut, Albert-Ludwigs-Universität Freiburg, Hermann-Herder-Str. 3, \\ D-79104 Freiburg, Germany \\ ${ }^{5}$ EUCOR Centre for Quantum Science and Quantum Computing, \\ Albert-Ludwigs-Universitaet Freiburg, Hermann-Herder-Str. 3, 79104 Freiburg, Germany
}

\begin{abstract}
Recent works revealed that transmission of light beams carrying orbital-angular-momentum (OAM) through turbulence causes the optical vortex defining these beams to split into multiple vortices with unit topological charge. Here, we consider the numerical propagation of orbital-angular-momentum (OAM) modes through a horizontal atmospheric channel. By analysing the beam's phase front after transmission through turbulence, we confirm the occurence of vortex splitting, but we also witness the emergence of vortex-antivortex pairs. Moreover, by performing performing a decomposition of the transmitted wave into OAM modes, we show that while adaptive optics cannot cancel vortex splitting, it still is pretty efficient in diminishing the turbulence-induced crosstalk between different OAM modes.
\end{abstract}

Keywords: orbital angular momentum, vortex splitting, adaptive optics

\section{INTRODUCTION}

High-dimensional states of light bear the possibility of increasing the information capacity and the security of free-space quantum and classical communication. ${ }^{1}$ Spanning an unbounded space, the orbital angular momentum $(\mathrm{OAM})^{2}$ of light can be used to realize such high dimensional states. The phase front of a light beam carrying an OAM $\hbar \ell$ (twisted light) is characterised by an optical vortex with topological charge $\ell$ on the beam axis. ${ }^{3}$ Such a peculiar phase front is however very fragile with respect to turbulence-induced distortions. Hence, it is mandatory to resort to compensation techniques - such as adaptive optics $(\mathrm{AO})^{4,5}$ - in order to mitigate these effects.

In a recent experiment on the propagation of OAM-carrying beams over a 1.5-km-long intra-city free space link, ${ }^{6}$ and in subsequent theoretical work, ${ }^{7}$ it was suggested that the most detrimental turbulence-induced distortion on twisted light is vortex splitting. By vortex splitting, we intend the separation of an optical vortex of charge $\ell$, as the one characterizing the phase profile of an optical mode with OAM $\hbar \ell$, into $\ell$ vortices with unit topological charge. ${ }^{8,9}$ It was also claimed that traditional AO cannot compensate for vortex splitting, and therefore it is of little help for OAM based free-space communication. The aim of this contribution is to ponder the potential usefulness of $\mathrm{AO}$, its shortcomings notwithstanding.

For this goal, we numerically propagate OAM modes through a horizontal atmospheric channel which is similar to the one considered previously. ${ }^{6}$ By analysing the beams' phase front after transmission through turbulence, we observe that vortex splitting not only occurs but also is often accompanied by the emergence of vortex-antivortex pairs. Finally, we consider AO corrections from the fundamental point of view. Thereby, we exclude from our analysis practical aspects of retrieving the phase of an optical field containing vortices. ${ }^{10}$ By

Send correspondence to giacomo.sorelli@lkb.upmc.fr 
means of an OAM-mode decomposition of the transmitted wave, we show that while AO cannot cancel vortex splitting, it can be pretty efficient in diminishing turbulence-induced crosstalk between different OAM modes. Given that the latter is the most detrimental effect for quantum and classical communication, we conclude that despite vortex splitting, we should not dismiss the benefit of AO in free-space OAM-based communication.

\section{METHODS}

We consider a light beam with wavelength $\lambda=809 \mathrm{~nm}$, whose spatial profile is given by a Laguerre-Gaussian (LG) mode ${ }^{11}$ with azimuthal index $l_{0}=3$, radial index $p=0$ and beam waist $w_{0}=1.5 \mathrm{~cm}$. For comparison with the experimental results, ${ }^{6}$ we propagate the above mentioned LG mode through a turbulent channel of length $z=1500 \mathrm{~m}$ and analyse the received light through a circular aperture of diameter $15 \mathrm{~cm}$.

The transverse length-scale of phase fluctuations in a turbulent channel can be characterized by the Fried parameter $r_{0}=\left(0.423 k^{2} C_{n}^{2} z\right)^{3 / 5}$, with $k=2 \pi / \lambda$ the wave number, and $C_{n}^{2}$ the turbulence structure constant. ${ }^{12,13}$ When this length scale is much smaller than the typical size of the beam, then propagation will significantly distort the light's spatial profile. Conversely, when this length is much larger than the beam size, then the beam will experience only weak distortions. Accordingly, as a measure of the turbulence strength in our channel, we consider the ratio between the size of our beam and the Fried parameter: $W=w_{0} / r_{0}$. Another important parameter is the Rytov variance $\sigma_{R}^{2}=1.23 C_{n}^{2} k^{7 / 6} z^{11 / 6}$, which quantifies the amplitude of turbulence-induced intensity fluctuations, also known as scintillation. ${ }^{12,13}$ In particular, the Rytov variance allows to distinguish between the weak $\left(\sigma_{R}^{2}<1\right)$ and strong $\left(\sigma_{R}^{2}>1\right)$ scintillation regimes. ${ }^{12,13}$

For sufficiently short propagation distances, we are always in the weak scintillation regime, where turbulence distortions are dominated by phase fluctuations. ${ }^{12,13}$ We exploit this fact to simulate light propagation through turbulence with a multiple-phase-screen approach. ${ }^{14,15}$ This method consists in splitting a turbulent channel into shorter segments where turbulence-induced phase distortions are introduced via random phase screens, while the evolution between two successive screens is described as free-space propagation in vacuum. In particular, we consider turbulence strengths up to $W=1.5$. The weak scintillation at each partial propagation step is verified by splitting our 1.5-km-long channel into 24 segments. We generate the random phase screens with Kolmogorov statistics, defined by the phase power spectral density $\Phi(\kappa)=0.49 r_{0}^{-5 / 3} \kappa^{-11 / 3}$, using a sub-harmonic method with seven sub-harmonics. ${ }^{14,16,17}$

To assess the efficiency of AO in presence of vortex splitting, we considered two different AO models (see also ${ }^{4,5}$ ), where phase distortions of a Gaussian beacon are measured and used to correct the LG mode. In the first model, we assume that the phase of the propagated beacon is fully reconstructed with spatial resolution limited only by our numerical grid. This corresponds to an ideal AO system, whose performance is experimentally unachievable. On the contrary, the second AO system only stabilizes the direction of the incoming beam with a tip-tilt mirror. Such a system only compensates for the two lowest-order distortions (tip and tilt) of atmospheric turbulence, which are however dominant in weak turbulence. ${ }^{18}$ By considering the simplest AO system, as well as its ideal counterpart, we can put a lower and an upper bound on the performance of any realistic AO system.

\section{RESULTS}

Let us start by observing how the phase of an OAM-carrying beam evolves upon propagation through the described above turbulent channel for a turbulence strength $W=0.5$. In Fig. 1, we see that already in the early stage of the propagation the original optical vortex, with topological charge $Q_{0}=3$, splits into three spatially separated vortices with charges $Q_{i}=1$. When the beam propagates further away from the sender, the vortices wander off the beam axis. Figure 1 also shows us that in addition to vortex splitting, turbulence induces the creation and annihilation of vortex-antivortex pairs, i.e., couples of vortices with opposite topological charge (in general, $Q= \pm 1$ ). The reader should also notice that the fine phase modulations visible in Fig. 1, especially in the first part of the propagations, are numerical artefacts which, however, occur in regions of zero intensity, and do not affect the successive propagation.

Higher-order vortex instability is well known in singular optics ${ }^{3}$ and it can be understood as follows. Vortices, as all field singularities, can only occur at points of vanishing intensity. Around these points, a general solution 

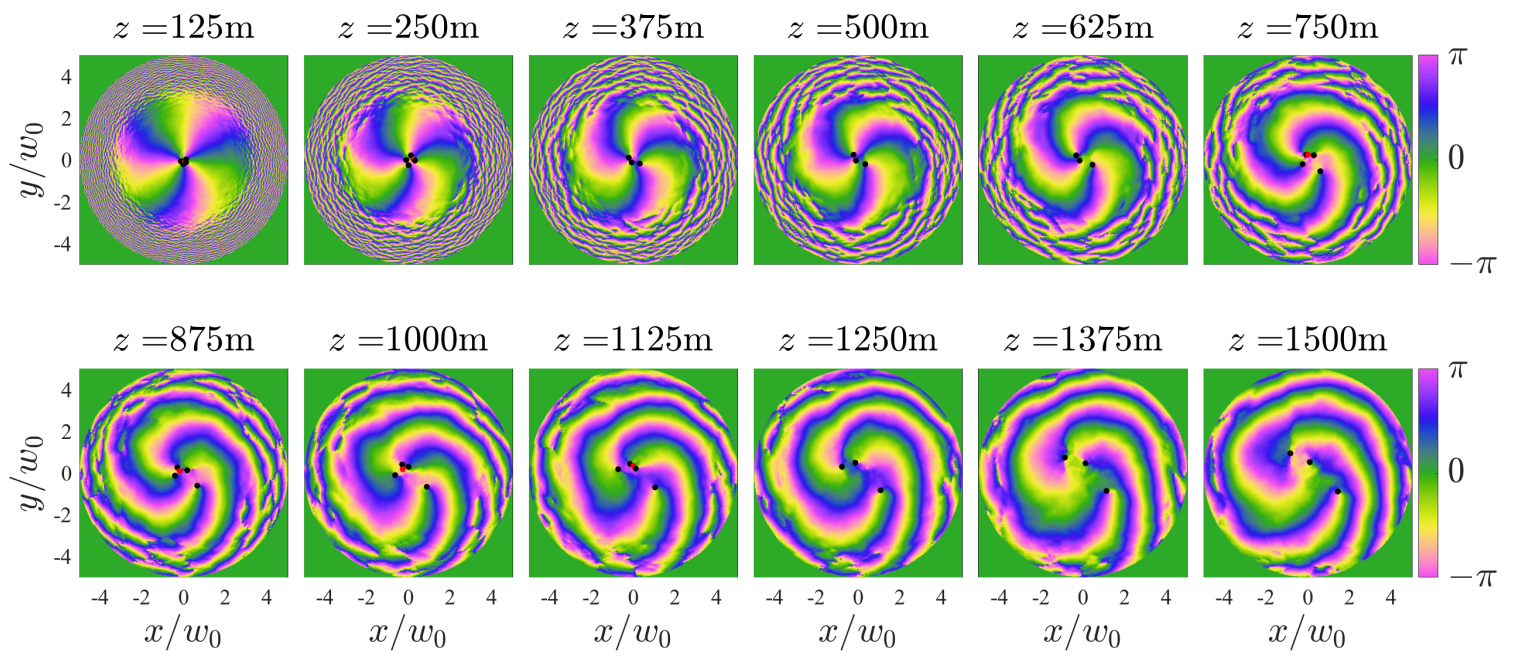

Figure 1. Phase profile of an $l_{0}=3 \mathrm{LG}$ mode transmitted through a turbulent channel (turbulence strength $W=0.5$ ) recorded every $125 \mathrm{~m}$ between the sender (at $z=0$ ) and the receiver (at $z=1500 \mathrm{~m}$ ). The dots indicate the location of optical vortices, with colour-encoded topological charge: black for $Q=+1$ and red for $Q=-1$.

of the wave equation featuring a vortex of charge $s$ can be written $\mathrm{as}^{3}$

$$
\psi(x, y)=a(x+i y)^{|s|}+b(x-i y)^{|s|}
$$

with $a$ and $b$ complex numbers, and $|a|>|b|$ for $s>0$ and $|a|<|b|$ for $s<0$. Equation (1) tells us that at the vortex position, the field $\psi$ and all its mixed partial derivatives up to order $|s|-1$ must vanish, while all mixed partial derivatives of order $s$ must be different from zero. Accordingly, $|s|(|s|+1)$ conditions must be exactly satisfied for an optical vortex of order $s$ to appear, and every perturbation of the field that breaks at least one of this conditions will result in vortex splitting. On the contrary, the sum of the topological charges of all vortices in an optical field,

$$
Q=\sum_{i=1}^{N_{v}} Q_{i},
$$

is a conserved quantity in optical propagation..$^{3,8,9}$ The combination of these two effects, higher-order vortex instability and conservation of $Q$, explain the behaviour observed in Fig. 1.

To quantify the amount of turbulence-induced vortex splitting, we use the vortex-splitting ratio $\mathcal{V}$, namely the average distance of the vortices from the beam axis divided by the beam radius. Such a quantity was introduced by Lavery. ${ }^{7}$ We modified this definition by weighting the vortices' positions with their topological charges to take into account the creation and annihilation of vortex-antivortex pairs. Formally, we can write the vortex-splitting ratio as

$$
\mathcal{V}=\frac{1}{N_{v} w_{0}} \sum_{i=1}^{N_{v}} Q_{i} \rho_{v}^{(i)},
$$

where for each vortex, identified by the index $i, \rho_{v}^{(i)}$ is the distance from the beam axis, $Q_{i}$ is the topological charge, $N_{v}$ is the total number of vortices in the phase profile, and $w_{0}$ is the beam waist.

The left panel of Fig. 2 shows the evolution of the vortex splitting ratio $\mathcal{V}$ with the propagation distance $z$ for different turbulence strengths. These results show a monotonic growth of $\mathcal{V}$ with a rate that increases with the turbulence strength. The apparent slow-down of the vortex-splitting observed for $W=1.5$ close to the receiving plane (purple diamond in the left panel of Fig. 2) is due to the degrading performance of our vortex search routine at large turbulence strengths. ${ }^{19}$ Our results are in qualitative agreement with those of Lavery. ${ }^{7}$ However, in the latter case $^{7}$ the vortex-splitting ratio grows slower with the propagation distance. This stems from the different numerical simulation strategy, ${ }^{7}$ which underestimate the turbulence strength in the channel. 

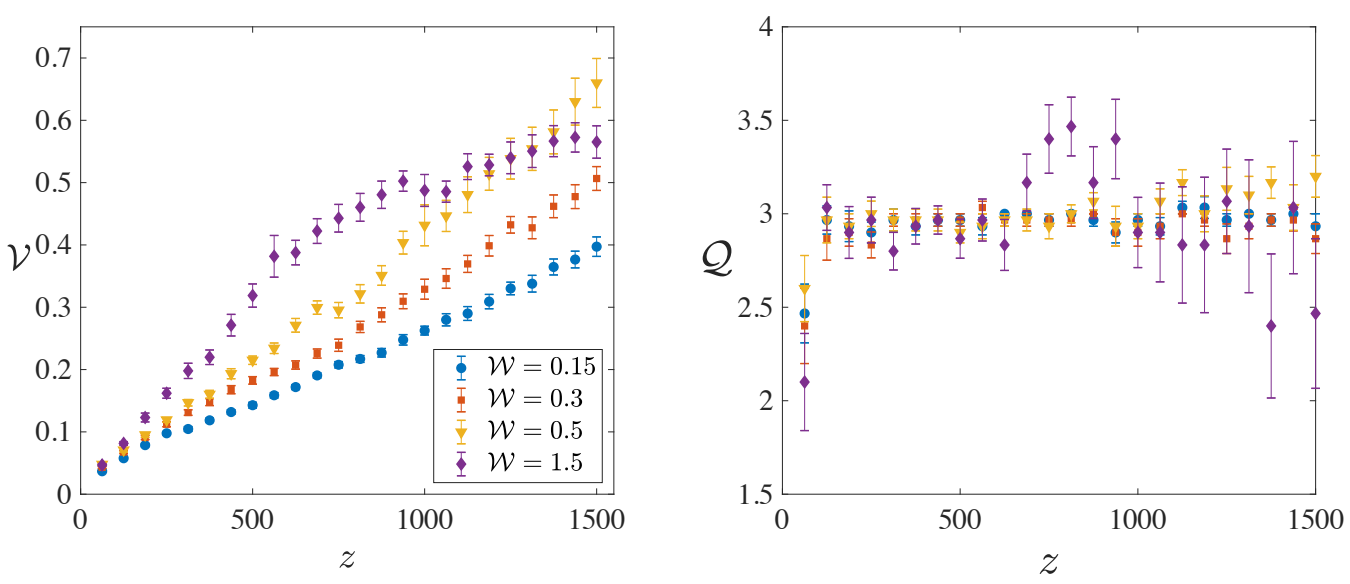

Figure 2. (Left) Vortex-splitting ratio $\mathcal{V}$ (see (3)) as a function of the propagation distance $z$ for different turbulence strengths $W$. (Right) Total topological charge $Q$ (see (2)) plotted against the propagation distance $z$ for the same values of the turbulence strengths $W$ as in the left plot. Both panels result from the propagation of an LG mode $\left(l_{0}=3, p=0\right)$ averaged over 30 random realizations of the turbulent channel. Error bars are standard deviations from the mean.

In the right panel of Fig. 2, we present the sum $Q$ (see (2)) of all topological charges in the phase profile. As discussed above, the total charge $Q$ must be a conserved quantity. We see that the total charge is correctly predicted in most cases, however, it deviates from its expected value $Q=3$ for short propagation distances, as well as high turbulence strengths.

The underestimation of $Q$ that we observe for $z=125 \mathrm{~m}$ is a simple spatial resolution issue. In fact, so close to the sender, even though the vortex splitting already happened, the vortices are still not well-separated (see the top left panel in Fig. 1), and our detection algorithm is unable to distinguish them. However, these unresolved vortices do not critically influence the estimation of the small vortex-splitting ratio close to the sender.

For larger turbulence strengths, there are two effects that may lead to an incorrect determination of the total topological charge. First, to reduce the sensitivity of our results with respect to numerical noise at the boundary of the aperture (where the amplitude should vanish, but may be garnished by finite values of the order of the numerical precision), we search for vortices only in a finite region around the beam axis. For large turbulence strengths, it may happen that a vortex wanders away from this region and therefore remains undetected. This physical effect causes underestimation of the vortex-splitting ratio and of the total topological charge. Second, at larger turbulence strengths, the phase acquires finer spatial structures which are difficult to resolve numerically and which lead to failures of the vortex detection routine. ${ }^{19}$ Numerical errors may result in both under- and overestimation of the total topological charge (see Fig. 2).

The above discussion proved that our numerical routine is able to correctly describe vortex splitting within a given range of turbulence conditions. We now aim at analysing how this phenomenon affects the capability of AO to protect light beams carrying OAM. A previous work ${ }^{7}$ considered this problem and concluded that AO cannot be efficient in presence of vortex splitting. However, we believe that the poor AO-performance observed in this work $^{7}$ is mainly due to the unfavorable diffraction conditions that he assumed and not by vortex splitting. To support this point, we slightly modified our simulation parameters to mitigate diffraction effects. In particular, we increased the beam waist to $w_{0}=0.03 \mathrm{~m}$, and the receiver aperture to $0.2 \mathrm{~m}$, leaving all other parameters unchanged.

Our results, for a turbulence strength $W=1$, are plotted in Fig. 3. The first row in Fig. 3 shows the stability of the intensity ring of the LG modes that we discussed in previous work ${ }^{5}$ and which was observed experimentally. ${ }^{20}$ Let us have a closer look at the phase distributions. In the uncorrected case (panel (d)), we observe a highly distorted phase profile where the three vortices originating from the splitting of the original $Q=3$ vortex are accompanied by two vortex-antivortex pairs. Tip-tilt corrections (panel (e)) do not significantly reduce the phase distortions, and leave the vortices unaltered. In panel (f), we see that, as expected, also the ideal correction does not modify the positions of the vortices, but it significantly modifies the phase distribution 

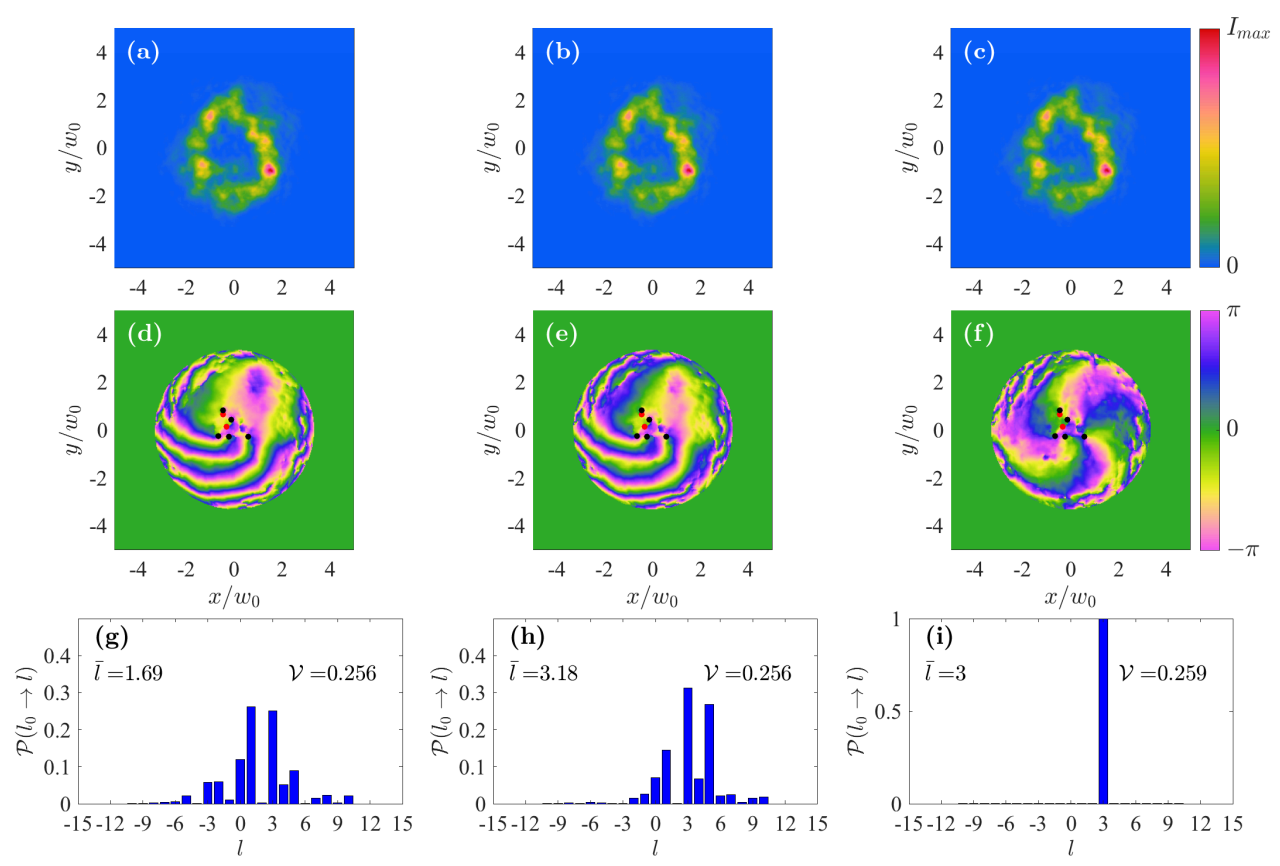

Figure 3. (a-c) Intensity and (d-f) phase distributions of an LG mode with azimuthal index $l_{0}=3$ and radial index $p=0$ transmitted through a turbulent channel with turbulence strength $W=1$. The dots in the phase panels represent the positions of the optical vortices with color-coded topological charge: black for $Q=+1$ and red for $Q=-1$. Panels (g-i) show a decomposition of the received field into $p=0$ LG modes averaged over 100 realizations of the turbulence channel together with the average OAM (in units of $\hbar$ ) $\bar{l}$ of the distribution, and the average vortex splitting ratio $\mathcal{V}$. The different columns correspond to different degrees of AO corrections: panels (a), (d), (g) no correction, (b), (e), (h) tip-tilt correction, (c), (f), (i) ideal AO.

so that it closely resembles the one of a mode which propagated in the absence of perturbations. The observation that for an ideally corrected beam both intensity and phase resemble those of an unperturbed LG mode suggests that $\mathrm{AO}$ can still provide some benefits, despite vortex splitting.

To make this conclusion more quantitative, we decomposed the propagated field in the basis of LG modes with $p=0$. How the intensity of the input $l_{0}=3 \mathrm{LG}$ mode spreads over this basis is plotted in panels (g-i) of Fig. 3, displaying the probability distribution $\mathcal{P}\left(l_{0} \rightarrow l\right)$ of finding the output field in the LG mode with the azimuthal index $l$. Comparing the different panels, we see that already tip-tilt corrections (panel (h)) mitigate the spreading of the OAM distribution by depleting the populations of those modes which are further away from the input modes. When using ideal AO (panel (i)) the populations of modes different from the input one, are substantially negligible. We also see that the average OAM of the distribution is significantly lower than the one of the input mode $\left(l_{0}=3\right)$, while it is close to this value in the tip-tilt corrected case and is exactly $\bar{l}=3$ in the ideally corrected one. We point out that this shift of the mean OAM towards a lower value, which cannot be reproduced by weak turbulence models (e.g. single phase screen models ${ }^{21-23}$ ), was the experimental observation ${ }^{6}$ that triggered the investigation of vortex splitting. ${ }^{7}$ Finally, in panels $(\mathrm{g}-\mathrm{i})$ of Fig. 3, we also included the average vortex splitting ratio, and we see that it is substantially unaffected by the corrections*. This confirms our idea that $\mathrm{AO}$ can be very efficient in correcting OAM modes even in the presence of vortex splitting.

\section{CONCLUSIONS}

We investigated the impact of vortex splitting on the efficiency of AO protection of OAM modes propagated through atmospheric turbulence. We proved that our numerical simulation routine can accurately reproduce the monotonic increase of vortex splitting with the propagation distance and the turbulence strength. ${ }^{7}$ It was also

\footnotetext{
${ }^{*}$ The slightly different value in the ideally corrected case can be imputed to numerical noise.
} 
suggested $^{7}$ that given its monotonicity, the vortex splitting ratio (3) could be an appropriate measure to quantify the turbulence-induced distortions of OAM beams. Although this idea deserves further investigations, our results show that vortex splitting is accompanied by the generation of vortex-antivortex pairs, which wander through the phase profile and are not distinguishable from the vortices originating from the splitting of a higher-order vortex. These pairs of vortices with opposite topological charge may affect the monotonicity of the vortex-splitting under strong turbulence. Ultimately, we observed that - even in presence of vortex splitting - AO remains capable of mitigating turbulence-induced crosstalk between different OAM modes, which is the most detrimental effect for quantum and classical communication applications.

\section{Acknowledgements}

VS acknowledges support by the Strategiefonds der Albert-Ludwigs-Universitaet Freiburg.

\section{REFERENCES}

[1] N. J. Cerf, M. Bourennane, A. Karlsson, and N. Gisin, "Security of quantum key distribution using $d$-level systems," Phys. Rev. Lett. 88, p. 127902, Mar 2002.

[2] L. Allen, M. W. Beijersbergen, R. J. C. Spreeuw, and J. P. Woerdman, "Orbital angular momentum of light and the transformation of Laguerre-Gaussian laser modes," Phys. Rev. A 45, pp. 8185-8189, Jun 1992.

[3] M. Dennis, K. O'Holleran, and M. Padgett, "Singular optics: optical vortices and polarization singularities," Progress in Optics 53, pp. 293-363, 2009.

[4] N. Leonhard, G. Sorelli, V. N. Shatokhin, C. Reinlein, and A. Buchleitner, "Protecting the entanglement of twisted photons by adaptive optics," Phys. Rev. A 97, p. 012321, Jan 2018.

[5] G. Sorelli, N. Leonhard, V. N. Shatokhin, C. Reinlein, and A. Buchleitner, "Entanglement protection of high-dimensional states by adaptive optics," New Journal of Physics 21, p. 023003, feb 2019.

[6] M. P. J. Lavery, C. Peuntinger, K. Günthner, P. Banzer, D. Elser, R. W. Boyd, M. J. Padgett, C. Marquardt, and G. Leuchs, "Free-space propagation of high-dimensional structured optical fields in an urban environment," Science Advances 3(10), 2017.

[7] M. P. J. Lavery, "Vortex instability in turbulent free-space propagation," New Journal of Physics 20, p. 043023, apr 2018.

[8] G. Gbur and R. K. Tyson, "Vortex beam propagation through atmospheric turbulence and topological charge conservation," J. Opt. Soc. Am. A 25, pp. 225-230, Jan 2008.

[9] X.-L. Ge, B.-Y. Wang, and C.-S. Guo, "Evolution of phase singularities of vortex beams propagating in atmospheric turbulence," J. Opt. Soc. Am. A 32, pp. 837-842, May 2015.

[10] D. L. Fried, "Branch point problem in adaptive optics," J. Opt. Soc. Am. A 15, pp. 2759-2768, Oct 1998.

[11] A. E. Siegman, Lasers, University Science Books, 1986.

[12] A. Ishimaru, Wave Propagation and Scattering in Random Media, An IEEE OUP classic reissue, Wiley, 1999.

[13] L. Andrews and R. Phillips, Laser Beam Propagation Through Random Media, SPIE Press monograph, Society of Photo Optical, 2005.

[14] J. D. Schmidt, Numerical Simulation of Optical Wave Propagation with examples in MATLAB, SPIE Press, Bellingham, Washington 98227-0010 USA, 2010.

[15] V. P. Lukin and B. V. Fortes, Adaptive beaming and imaging in the turbulent atmosphere, SPIE Press, 2002.

[16] R. G. Lane, A. Glindemann, and J. C. Dainty, "Simulation of a kolmogorov phase screen," Waves in Random Media 2(3), pp. 209-224, 1992.

[17] E. M. Johansson and D. T. Gavel, "Simulation of stellar speckle imaging," Proc.SPIE 2200, pp. 2200 $2200-12,1994$.

[18] R. Tyson, Principles of Adaptive Optics, Series in Optics and Optoelectronics, CRC Press, 2010.

[19] G. Sorelli, Quantum state transfer in diffractive and refractive media, Dissertation, Albert-LudwigsUniversitaet Freiburg, DOI: 10.6094/UNIFR/150962, 2019. 
[20] M. Krenn, J. Handsteiner, M. Fink, R. Fickler, R. Ursin, M. Malik, and A. Zeilinger, "Twisted light transmission over 143 km," Proceedings of the National Academy of Sciences 113(48), pp. 13648-13653, 2016.

[21] C. Paterson, "Atmospheric turbulence and orbital angular momentum of single photons for optical communication," Phys. Rev. Lett. 94, p. 153901, Apr 2005.

[22] G. A. Tyler and R. W. Boyd, "Influence of atmospheric turbulence on the propagation of quantum states of light carrying orbital angular momentum," Optics letters 34(2), pp. 142-144, 2009.

[23] N. D. Leonhard, V. N. Shatokhin, and A. Buchleitner, "Universal entanglement decay of photonic-orbitalangular-momentum qubit states in atmospheric turbulence," Phys. Rev. A 91, p. 012345, Jan 2015. 